\title{
COVID-19 associated coagulopathy is correlated with increased age and markers of inflammation response
}

\author{
Laurentiu Stratan ${ }^{1,2}$, Catalin Tiliscan ${ }^{1,2 *}$, Victoria Arama ${ }^{2,3}$, Mihai Lazar ${ }^{1,4}$, \\ Angelica Visan ${ }^{3,5}$, Oana Ganea ${ }^{2}$, Maria I. Trifonescu ${ }^{2}$, Sorin S. Arama ${ }^{1,2}$, Daniela \\ Ion $^{1}$
}

1. Pathophysiology, Carol Davila University of Medicine and Pharmacy, Romania

2. Adulti 3 Dept, Prof. Dr. Matei Bals National Institute of Infectious Diseases, Romania

3. Infectious Diseases, Carol Davila University of Medicine and Pharmacy, Romania

4. Radiology Dept, Prof. Dr. Matei Bals National Institute of Infectious Diseases, Romania

5. Copii 11 Dept, Prof. Dr. Matei Bals National Institute of Infectious Diseases, Romania

\begin{abstract}
Background: The severe manifestations of the coronavirus disease 2019 (COVID-19) are linked to viral hyperinflammation, cytokine release syndrome and subsequent coagulation disturbances. The most common coagulation abnormality observed in COVID-19 patients is the elevation of the plasma levels of D-dimers. The aim of this study was to evaluate the characteristics of COVID-19-associated inflammatory syndrome and coagulopathy, in correlation with disease severity. Methods: We performed a cross-sectional study, enrolling all consecutive COVID-19 patients treated in the Adulti 3 Department of the Prof. Dr. Matei Bals National Institute of Infectious Diseases, Bucharest, Romania, between 1st march and 30th September 2020. We recorded clinical and epidemiological characteristics, inflammatory markers, coagulation abnormalities and lymphocyte count. The severity of lung involvement was assessed using native Computed Tomography examination. Results: We included 106 patients with SARS-COV2 infection, 50 males (47.2\%) and 56 females (52.8\%), age range 14-91 years. All markers of inflammation were increased in our study in patients with severe disease, as were lactate dehydrogenase, monocyte distribution width, and neutrophil-to-lymphocyte ratio. An elevated level of serum D-dimers was observed in approximately half of our subjects and was associated with disease severity. Our best linear regression model for predicting COVID-19 coagulopathy (manifested as abnormal D-dimer levels) included age, fibrinogen, and lymphocyte count. Conclusion: Our findings emphasize the association between COVID-19 coagulopathy and the presence of systemic inflammation. A significant proportion of patients with moderate and severe disease had coagulation abnormalities and these were linked with the presence of inflammation and older age..
\end{abstract}

Keywords: inflammation, coagulopathy, COVID-19, SARS-CoV2

Received: $3^{\text {rd }}$ August 2021; Accepted: $8^{\text {th }}$ October 2021; Published: $12^{\text {th }}$ October 2021

\footnotetext{
* Corresponding author: Catalin Tiliscan, Pathophysiology, Carol Davila University of Medicine and Pharmacy, Bucharest, Romania. E-mail: catalin.tiliscan@gmail.com
} 


\section{Introduction}

In late 2019 a highly transmissible coronavirus named severe acute respiratory syndrome coronavirus 2 (SARS-COV-2) emerged, causing a pandemic of acute respiratory disease named "coronavirus disease 2019" (COVID-19), which still threatens human health and public safety at present (1).

COVID-19 can have various presentations, from mild and moderate disease to severe, rapidly progressing pneumonia associated with acute respiratory distress syndrome and multi-organ failure (2). The severe manifestations of this disease are linked to a condition determined by viral hyperinflammation: the cytokine storm characterized by elevated levels of different pro-inflammatory cytokines (3). Systemic inflammation can lead to the activation of the coagulation system, by tissue factor-mediated fibrin formation, mainly promoted by IL-6 (4). Along with virus specific effect on endothelial cells which ultimately increases the angiotensin II availability, promoting platelet adhesion and aggregation, systemic inflammation associated with moderate-severe forms of COVID-19 determines a procoagulant state and thrombotic events (5).

The most common coagulation abnormality observed in COVID-19 patients is an elevation of the plasma level of D-dimers, which is a risk factor for death in adult patients infected with SARS-CoV-2 (6). Other hemostatic defects seen in COVID-19 patients are prolonged prothrombin time (PT) and thrombocytopenia, possibly also associated with a poor outcome. COVID-19 coagulopathy was associated with an increase in the levels of procoagulant factors such as fibrinogen, as well as an increase in CRP levels, that are linked with a higher mortality rate (5).

Our aim was to evaluate the characteristics of COVID-19-associated inflammatory syndrome and coagulopathy, in correlation with disease severity.

\section{Materials and Methods}

The study enrolled all consecutive SARS-CoV-2 positive patients, treated in the Adulti 3 COVID department of the Prof. Dr. Matei Bals National Institute of Infectious Diseases, Bucharest, Romania, between 1st March and 30th September 2020.The study protocol was approved by the ethics committee of Prof. Dr. Matei Bals National Institute of Infectious Diseases (protocol no. C0408/2020). All patients had a RT-PCR rhinopharyngeal swab positive for SARS-CoV-2 infection. We selected all consecutive clinical records of patients over 14 years of age, treated in our department and recorded: age, gender, body mass index (BMI), previous clinical history, COVID-19 signs and symptoms, oxygen therapy requirement, markers of inflammation (erythrocyte sedimentation rate (ESR), fibrinogen and C-reactive protein plasma levels - Siemens BN ProSpec System), transaminases (alanine aminotransferase - ALT and aspartate aminotransferase - AST), lactate dehydrogenase (LDH), myoglobin, creatine kinase (CK), creatine kinase $\mathrm{MB}$ (CK-MB), N-terminal (NT)-pro hormone BNP (NT-proBNP) (VITROS 5.1 FS, VITROS 4600, Siemens Dimension EXL Chemistry Analyzer, RAMP 200), complete blood count, neutrophils/ lymphocytes ratio (Ne/Ly), Monocyte Distribution Width (MDW) - (DXH 900, ADVIA 2120, ADVIA 2120i, Nihon Kohden Celltac F MEK8222 ) and markers of coagulopathy (aPTT, prothrombin ratio, D-dimer plasma levels) - (Sysmex CS-2000i, ACL TOP 500, Grifols QNext Hemostasis Analyzer).

The severity of the lung involvement was assessed using native Computed Tomography (CT) examination. We performed spiral acquisitions (with breath-hold when possible) with a pitch of 1.2, CARE Dose4D and CARE $\mathrm{kV}$ active in order to reduce the radiation dose, $1.2 \mathrm{~mm}$ colimmation and $3 \mathrm{~mm}$ reconstructions in both mediastin (B31f image filter) and lung window 
(B80f ultra sharp image filter). For the lung involvement staging we used the following score:

- 1 point for each lung segment involved

- 3 points if the lesions occurred in both lungs

- 3 points if alveolar involvement was detected

- 2 points if pleurisy or pericarditis were present

We considered a mild pneumonia for a score lower than 9, a moderate pneumonia for a score between 9 and 18, and severe for a score over 18 . We presented the variables with normal distribution as means $(+/-$ standard deviation [SD]) and we used median (interquartile range) for non-gaussian continuous data. Independent-sample t-test was used to assess the differences in means between groups with normal distribution. We computed $95 \%$ confidence intervals for differences between means. Mann-Whitney test was used for non-parametric continuous variables. In order to test for statistical significance for categorical variables, we used Chi-square or Fisher's exact test and Odds ratio (OR) was computed. Linear regression models were fitted to test the association of coagulopathy with continuous variables, included in the univariate and multivariate models either because of their known or suspected association with COVID-19 associated coagulopathy. Variables with a $\mathrm{p}$ value of $\leq 0.1$ in the univariate models were included in the multivariate regression analysis. The correlation coefficient (R) was used to evaluate the linear correlation between dependent and independent variables. Two-tailed p-values. Statistical significance was set at 5\%. All analyses were performed with SPSS ${ }$ Statistics v.22.0, IBM $\AA$, New York, USA.

\section{Results}

We included 106 consecutive patients with SARS-COV2 infection, 50 males (47.2\%) and 56 females (52.8\%), admitted in the Adulti 3 Department. The age range for the entire group was $14-91$ years, with a mean age of $49.6 \pm 17.8$ years
$(46.1 \pm 16.9$ vs $52.7 \pm 18.1$ years males vs females, $\mathrm{p}=0.03)$. Fourteen patients (13.2\%) were asymptomatic, and $92(86.7 \%)$ had varying degrees of signs and symptoms, from mild severity to severe respiratory distress.

Based on radiological imaging findings, 11 patients $(10.3 \%)$ had no involvement, 30 (28.8\%) patients had mild disease, 43 (40.5\%) had moderate and $22(20.7 \%)$ had severe disease. We divided the patients into two subgroups: G1 included moderate and severe disease and G2 patients with mild or no pulmonary lesions. Thirty-six patients (including all patients with severe disease and 14 with moderate disease) had acute hypoxemic respiratory failure that required oxygen therapy.

Table I presents subject characteristics stratified by pulmonary disease severity. The following variables presented statistically significant differences in the univariate analysis between the two groups: age, BMI, the presence of hypertension and diabetes mellitus, Ne/Ly, MDW, ESR, fibrinogen, CRP, blood urea, AST, ALT, D-dimers and LDH. G1 patients were older $(53.3 \pm 15.4$ vs $43.7 \pm 19.7$ years, $\mathrm{p}=0.00)$, had a higher mean BMI $(29.8 \pm 7.3$ vs $25.5 \pm 4.6 \mathrm{~kg} /$ $\left.\mathrm{m}^{2}, \mathrm{p}=0.00\right)$, were more likely to be obese $(43 \%$ vs $12.2 \%, \mathrm{p}=0.00)$, hypertensive ( $40 \%$ vs $17 \%$, $\mathrm{p}=0.04)$ and have diabetes mellitus $(24.6 \%$ vs $2.4 \%, \mathrm{p}=0.01$ ), when compared to patients with mild disease.

Higher Ne/Ly (3.7 vs 2.2, p=0.00), MDW (24.5 \pm 3.4 vs $20.7 \pm 2.9, p=0.00)$, plasma fibrinogen $(522.67 \pm 162.9$ vs $343.1 \pm 83.5 \mathrm{mg} /$ $\mathrm{dl}, \mathrm{p}=0.00), \quad \operatorname{ESR}(45.3 \pm 24$ vs $18 \pm 14.2 \mathrm{~mm} / \mathrm{h}$, $\mathrm{p}=0.00)$, CRP (31.7 vs $1.9 \mathrm{mg} / \mathrm{L}, \mathrm{p}=0.00)$, blood urea $(39.7 \pm 16.6$ vs $33.6 \pm 11.6, p=0.04)$, ALT (32.5 vs $25 \mathrm{UI} / \mathrm{L}, \mathrm{p}=0.04)$, AST (37 vs $30 \mathrm{UI} / \mathrm{L}$, $\mathrm{p}=0.00)$, D-dimers (232 vs $133 \mathrm{mg} / \mathrm{L}, \mathrm{p}=0.01)$ and $\mathrm{LDH}(327 \pm 128.11$ vs $209.3 \pm 64.85 \mathrm{UI} / \mathrm{L}$, $\mathrm{p}=0.00$ ) were observed in patients with moderate/severe pulmonary disease, compared to G2 subjects. 
Table I. Epidemiological, clinical and biological characteristics of study patients by pulmonary disease severity.

\begin{tabular}{|c|c|c|c|c|}
\hline Characteristics & Total patients & $\begin{array}{c}\text { Patients with } \\
\text { moderate-severe } \\
\text { disease (G1) }\end{array}$ & $\begin{array}{c}\text { Patients with mild } \\
\text { disease or no pul- } \\
\text { monary lesions } \\
\text { (G2) }\end{array}$ & $\mathrm{P}$ \\
\hline $\mathrm{N}$ & 106 & 65 & 41 & \\
\hline Age(years) & $49.6 \pm 17.8$ & $53.3 \pm 15.4$ & $43.7 \pm 19.7$ & $0.00^{*}$ \\
\hline Male & $50(47.2)$ & $33(66)$ & $17(34)$ & 0.35 \\
\hline Obesity & $33(31.1)$ & $28(43)$ & $5(12.2)$ & $0.00^{*}$ \\
\hline BMI(kg/m2) & $28.2 \pm 6.7$ & $29.8 \pm 7.3$ & $25.5 \pm 4.6$ & $0.00^{*}$ \\
\hline Hypertension ${ }^{1}$ & $33(31.1)$ & $26(40)$ & $7(17)$ & $0.04 *$ \\
\hline Active smoker & $10(9.4)$ & $5(7.7)$ & $5(12.2)$ & 0.73 \\
\hline Diabetes Mellitus & $17(16)$ & $16(24.6)$ & $1(2.4)$ & $0.01 *$ \\
\hline Leukocyte count(cells/mm3) & $6991.2 \pm 2725.3$ & $7360.3 \pm 293$ & $6391.5 \pm 2252$ & 0.07 \\
\hline Lymphocyte count(cells/mm3) & $1529.2 \pm 898.1$ & $1433.5 \pm 1026.8$ & $1682.2 \pm 622.9$ & 0.17 \\
\hline Neutrophil count(cells/mm3) & $4861.6 \pm 2337.1$ & $5346.2 \pm 2513$ & $4086.2 \pm 1795$ & $0.00^{*}$ \\
\hline Ne/Ly(neutrophils/lymphocytes ratio) ${ }^{\mathrm{a}}$ & $3(3.5)$ & $3.7(5.9)$ & $2.2(1.6)$ & $0.00^{*}$ \\
\hline Haemoglobin(g/dl) & $13.9 \pm 1.5$ & $13.94 \pm 1.5$ & $13.80 \pm 1.6$ & 0.64 \\
\hline Platelets count (cells/mm3) & $222913.1 \pm 91706.2$ & $225306.2 \pm 96893.9$ & $219084 \pm 83789$ & 0.73 \\
\hline MDW & $23 \pm 3.7$ & $24.5 \pm 3.4$ & $20.7 \pm 2.9$ & $0.00^{*}$ \\
\hline Fibrinogen (mg/dl) & $453 \pm 163$ & $522.67 \pm 162.9$ & $343.1 \pm 83.5$ & $0.00^{*}$ \\
\hline ESR $(\mathbf{m m} / \mathbf{h})$ & $33.9 \pm 24.6$ & $45.3 \pm 24$ & $18 \pm 14.2$ & $0.00^{*}$ \\
\hline $\operatorname{CRP}(g /)^{a}$ & $15.8(59.6)$ & $31.7(84.7)$ & $1.9(7.6)$ & $0.00^{*}$ \\
\hline Glucose (mg/dl) & $118 \pm 55.1$ & $132 \pm 65.9$ & $97.23 \pm 17.1$ & $0.00^{*}$ \\
\hline Blood urea (mg/dl) & $37.3 \pm 15.1$ & $39.7 \pm 16.6$ & $33.6 \pm 11.6$ & $0.04 *$ \\
\hline Creatinine (mg/dl) & $0.7 \pm 0.2$ & $0.8 \pm 0.2$ & $0.7 \pm 0.2$ & 0.17 \\
\hline ALT (UI/I) & $29(24)$ & $32.5(23)$ & $25(14)$ & $0.01^{*}$ \\
\hline $\operatorname{AST}\left(\mathbf{U I} / \mathbf{l}^{\mathrm{a}}\right.$ & $33.5(20)$ & $37(19)$ & $30(14)$ & $0.00^{*}$ \\
\hline Sodium $(\mathrm{mmol} / \mathrm{l})$ & $138.1 \pm 2.6$ & $137.51 \pm 2.8$ & $139.03 \pm 2$ & $0.00^{*}$ \\
\hline Potassium (mmol/l) & $4.1 \pm 0.4$ & $4.2 \pm 0.5$ & $4 \pm 0.3$ & $0.05^{*}$ \\
\hline aPTT (s) & $33.1 \pm 7.7$ & $33.5 \pm 8.5$ & $32.6 \pm 6.1$ & 0.63 \\
\hline Prothrombin Ratio (\%) & $85 \pm 15.8$ & $86.2 \pm 16.2$ & $87.9 \pm 15.4$ & 0.61 \\
\hline D-dimers $(\mathrm{mg} / \mathrm{l})^{\mathrm{a}}$ & $209.5(209.5)$ & $232(247.5)$ & $133(146)$ & $0.01 *$ \\
\hline NT-proBNP(ng/l) ${ }^{\text {a }}$ & $23.5(67)$ & $28(182)$ & $15(39)$ & 0.31 \\
\hline Troponin I (ng/ml) & $0.04 \pm 0.06$ & $0.05 \pm 0.07$ & 0.03 & 0.14 \\
\hline Myoglobin (ng/ml) ${ }^{a}$ & $68(90.6)$ & $89.4(114.9)$ & $44.8(52.4)$ & 0.06 \\
\hline CK (UI/I) $)^{\mathrm{a}}$ & $74(85)$ & $73.5(97)$ & $83(60)$ & 0.93 \\
\hline CK-MB (U/I) ${ }^{\mathrm{a}}$ & $9.5(8)$ & $9(10)$ & $9.5(7)$ & 0.83 \\
\hline LDH (UI/I) & $248 \pm 122.5$ & $327 \pm 128.11$ & $209.3 \pm 64.85$ & $0.00^{*}$ \\
\hline
\end{tabular}

An elevated D-dimers plasma level was observed in $52 \%$ of the total cases, and the presence of an abnormal D-dimer level was significantly associated to moderate/severe disease, with an OR of 2.38 (1.4 - 4.05, 95\% confidence interval, $\mathrm{p}=0.00)$. We observed no correlation between D-dimers abnormality and $\operatorname{sex}(p=0.7)$, but the patients with this coagulopathy were signifi- 
cantly older ( $57.2 \pm 17.6$ vs $41.3 \pm 13.7, \mathrm{p}=0.00)$, compared to the subjects with normal level of D-dimers.

A forward selection stepwise linear regression analysis was performed in order to obtain the most statistically significant model that predicted the plasma levels of D-dimers. In the univariate linear models the following independent variables were significantly correlated and predicted D-dimer plasma levels: age $(\mathrm{R}=0.28$, $\mathrm{p}=0.00)$ and fibrinogen $(\mathrm{R}=0.2, \mathrm{p}=0.04)$. The independent variables with a $p$ value of $\leq 0.1$ in the univariate analysis were included in the multivariate regression models: lymphocyte count $(\mathrm{R}=0.18, \mathrm{p}=0.06), \mathrm{CRP}(\mathrm{R}=0.18, \mathrm{p}=0.07)$ and $\mathrm{LDH}(\mathrm{R}=0.16, \mathrm{p}=0.1)$. The best multivariate regression model that predicted the plasma level of D-dimers included three independent variables: age, lymphocyte count and $\mathrm{LDH}(\mathrm{R}=0.42$, $\mathrm{R}$ square $=0.18$, adjusted $\mathrm{R}$ square $=0.15 .2$, $\mathrm{p}=0.00$ ). All the independent variables in the model presented a positive correlation with the plasma level of D-dimers.

\section{Discussions}

An elevated level of plasma D-dimers was observed in approximately half of our subjects, and was associated with disease severity. The presence of moderate or severe pulmonary lesions was associated with higher levels of plasma D-dimers, without other modifications of the studied coagulation tests.

SARS-CoV2-induced coagulopathy is correlated with a pro-coagulant state, pulmonary thromboembolism, thrombotic microangiopathy and with endotheliitis and microvascular dysfunction in the cardiovascular system (7). D-dimers elevations are the most frequent coagulopathy feature observed in COVID-19 patients, and are associated with disease severity. D-dimers represent a good biomarker indicative for disease severity (8).
Other coagulation abnormalities reported by other authors, such as prolongation of prothrombin time and a decrease of platelet count, were not found in our study (9).

All markers of inflammation (ESR, fibrinogen and CRP) were increased in our study in patients with severe disease, as were LDH, MDW and $\mathrm{Ne} /$ Ly. Interestingly, we observed moderate, but statistically significant increases of ALT, AST, and blood urea.

The best predictors of COVID-19 coagulopathy (manifested as abnormal D-dimer levels) were older age and increased fibrinogen. Our best linear regression model for predicting D-dimer levels included age, fibrinogen and lymphocyte count, all variables with a positive correlation. The correlation between D-dimers and fibrinogen (which is a marker of inflammation in COVID-19) observed in this study emphasizes the established connection between inflammation and coagulation. There are several proposed mechanisms that explain the link between inflammation and the presence of coagulopathy in COVID-19 patients.

Sars-CoV-2 infects human cells by targeting the angiotensin-converting enzyme 2 (ACE-2) receptors. These receptors are found on the surface of many types of cells, including epithelial and endothelial cells. The binding of SARS-CoV-2 to the endothelial cells by ACE-2 receptors can induce endothelial activation with the release of inflammatory cytokines (especially IL-1 and IL-6), which are responsible both for the activation of coagulation and inflammation frequently reported in COVID-19 patients (10). Systemic inflammation triggers the extrinsic pathway of coagulation, determining tissue factor-mediated thrombin generation (5). Among the pro-inflammatory cytokines involved in the process (cytokine-mediated coagulation activation), IL-6 appears to have a central role.

IL-6 plays an important role in haemostasis, exerting multiple effects on endothelial cells, leu- 
cocytes and hepatocytes, consecutively leading to synthesis of coagulation factors such as fibrinogen, tissue factor and factor VIII (11-13), as well as stimulation of platelet production. IL-6 can significantly increase fibrinogen synthesis both in the liver and in other sources, such as epithelial cells, which are similarly IL-6 responsive (14).

Lymphocytes also express ACE-2, and the bindnig of SARS-CoV-2 may induce lymphocyte apoptosis, which determines the characteristic lymphopenia observed in severe cases of COVID-19 (15). Neutrophils can also contribute to coagulation abnormalities, observed in COVID-19 patients. Barnes et al. found during autopsies of COVID-19 patients infiltration of neutrophils in pulmonary capillaries with acute capilaritis, the presence of neutrophils in the alveolar space and neutrophilic mucositis. These findings can explain why neutrophilia predicts poor outcomes in patients with COVID-19 (16), and the increase of neutrophil-to-lymphocyte ratio in severe cases (17), observed also in our study. Furthermore, the release of neutrophil extracellular traps (NETs) observed in COVID-19 patients can be involved in thromboinflammation by activating other prothrombotic pathways resulting in enhanced thrombin generation. Increasingly, NETs formation is recognized as a pathogenic mechanism linking coagulopathy and inflammation in multiple conditions (15).

SARS-CoV-2 binding of ACE-2 during acute infection may lead to the increase of plasma concentration of angiotensin II, which is normally metabolized to angiotensin by ACE-2 activity. Increased angiotensin may have further prothrombotic effects, by platelet and endothelial activation, vascoconstriction, and cytokine release (15).

Several patients' characteristics (such as advanced age and obesity) may participate to a hypercoagulable state. In our study, older age was a predictor for the level of plasma D-dimers, both in the univariate and multivariate analysis. The association between the risk of complications during viral respiratory infections and obesity was previously reported for influenza and for SARS and MERS, which share a significant genetic similarity with SARS-CoV-2 (80\% for SARS-CoV and 50\% with MERS-CoV) (18). We also report a significant correlation of obesity and increased BMI with the severity of disease, but not with the presence of coagulopathy. Similar to other studies, we report that COVID-19 pulmonary severity was associated also with increased age and the presence of hypertension and diabetes mellitus. We did not find a significant correlation between disease severity and male sex or lymphocyte count, as reported by other authors (19), but D-dimers alterations were associated with lymphocyte variations. Lymphopenia is frequently found in patients with severe forms of COVID-19. The responsible mechanisms are still not clearly known, but most researchers hypothesize that severe cases are associated with lymphocyte redistribution, sequestration in the lungs or bone marrow suppression via CD13 or CD66 (20-22).

\section{Conclusions}

Our study brings new data emphasizing the association of COVID-19 coagulopathy with the presence of systemic inflammation. A significant proportion of patients with moderate and severe disease present coagulation abnormalities and these are linked with the presence of inflammation and older age. Targeting the mechanisms underlying coagulopathy and inflammation may constitute new important therapeutic strategies for the treatment of this complex pathology.

\section{Abbreviations}

COVID-19 - "coronavirus disease 2019" SARS-COV-2 - severe acute respiratory syndrome coronavirus 2 
IL-6 - interleukin 6

IL-1 - interleukin 1

BMI - body mass index

ESR - erythrocyte sedimentation rate

CRP - C reactive protein

ALT - alanine aminotransferase

AST - aspartate aminotransferase

LDH - lactate dehydrogenase

CK - creatine kinase

CK-MB - creatine kinase muscle-brain

NT-pro-BNP - N-terminal pro-brain natriuretic peptide

Ne/Ly - neutrophils/lymphocytes ratio

MDW - monocyte distribution width

aPTT - activated partial thromboplastin time

$\mathrm{SD}$ - standard deviation

OR - odds ratio

ACE-2 - angiotensin-converting enzyme 2

NETs - neutrophil extracellular traps

CD13 - cluster of differentiation 13

CD66 - cluster of differentiation 66

\section{Acknowledgements}

The authors thank all patients for their contribution to the research, as well as all the medical staff involved in this study, especially senior and resident physicians for performing the COVID-19 data collection process, nurses, laboratory physicians, radiologists, epidemiologists for their involvement in recruiting and evaluation of the patients.

\section{Authors' contributions}

VA and CT conceived the design of the study, coordinated the research study group and drafted the manuscript. CT, LS and SSA performed statistical analyses and drafted the manuscript. AV, LS, ML, OG and MIT participated in patient enrollment and evaluation and follow-up and reviewed the literature. DI and AV reviewed the paper before submission. LS, CT, VA, ML, AV, OG, MIT, SSA and DA contributed equally to this work. All authors read and approved the final manuscript.

\section{Conflict of interest}

The authors declare that they have no competing interests. This paper received no funding, including internal support or grants from non-commercial institutions.

\section{References}

1. Hu B, Guo H, Zhou P, Shi ZL. Characteristics of SARSCoV-2 and COVID-19. Nat Rev Microbiol. 2021 Mar; 19(3):141-54. DOI: 10.1038/s41579-020-00459-7

2. Wu Z, McGoogan JM. Characteristics of and important lessons from the coronavirus disease 2019 (COVID-19) outbreak in China: summary of a report of 72314 cases from the chinese center for disease control and prevention. JAMA 2020;323:1239-42. DOI: 10.1001/ jama.2020.2648

3. Mehta P, McAuley DF, Brown M, Sanchez E, Tattersall RS, Manson JJ. COVID-19: consider cytokine storm syndromes and immunosuppression. Lancet 2020;395:1033-4. DOI: 10.1016/S01406736(20)30628-0

4. Levi M, van der Poll T. Inflammation and coagulation. Crit Care Med 2010;38:S26-34. DOI: 10.1097/CCM. 0b013e3181c98d21

5. Lazzaroni MG, Piantoni S, Masneri S, Garrafa E, Martini $G$, Tincani A, et al. Coagulation dysfunction in COVID-19: The interplay between inflammation, viral infection and the coagulation system. Blood Rev. 2021 Mar;46:100745. DOI: 10.1016/j.blre.2020.100745

6. Zhou F, Yu T, Du R, Fan G, Liu Y, Liu Z, et al. Clinical course and risk factors for mortality of adult inpatients with COVID19 in Wuhan, China: a retrospective cohort study. Lancet. 2020;395(10229):1054-62. DOI: 10.1016/S0140-6736(20)30566-3

7. Teuwen L, Geldhof V, Pasut A, Carmeliet P. COVID-19: the vasculature unleashed. Nature Rev Immunol. 2020;20:389-91. DOI: 10.1038/s41577-020-0343-0

8. Zhang L, Yan X, Fan Q, Liu H, Liu X, Liu Z, et al. D-dimer levels on admission to predict in-hospital mortality in patients with Covid-19. J Thromb Haemost. 2020;18:1324-9. DOI: 10.1111/jth.14859

9. Lee SG, Fralick M, Sholzberg M. Coagulopathy associated with COVID-19. CMAJ. 2020 May 25;192(21):E583. DOI: 10.1503/cmaj.200685

10. Connors JM, Levy JH. COVID-19 and its implications for thrombosis and anticoagulation. Blood. 2020 Jun 4;135(23):2033-40. DOI: 10.1182/blood.2020006000

11. Levi M, van der Poll T, Schultz M. Systemic versus localized coagulation activation contributing to organ 
failure in critically ill patients. Semin Immunopathol. 2012 Jan;34(1):167-79. DOI: 10.1007/s00281-0110283-7

12. Stouthard JM, Levi M, Hack CE, Veenhof CH, Romijn HA, Sauerwein HP, et al. Interleukin-6 stimulates coagulation, not fibrinolysis, in humans. Thromb Haemost. 1996 Nov;76(5):738-42. DOI: 10.1055/s-00381650653

13. Stirling D, Hannant WA, Ludlam CA. Transcriptional activation of the factor VIII gene in liver cell lines by interleukin-6. Thromb Haemost 1998;79:74-8. DOI: 10.1055/s-0037-1614223

14. Kerr R, Stirling D, Ludlam CA. Interleukin 6 and haemostasis. Br J Haematol. 2001 Oct;115(1):3-1. DOI: 10.1046/j.1365-2141.2001.03061.x

15. Leisman DE, Deutschman CS, Legrand M. Facing COVID-19 in the ICU: vascular dysfunction, thrombosis, and dysregulated inflammation. Intensive Care Med. 2020 Jun;46(6):1105-8. DOI: 10.1007/s00134020-06059-6

16. Wang D, Hu B, Hu C, Zhu F, Liu X, Zhang J, et al. Clinical Characteristics of 138 Hospitalized Patients With 2019 Novel Coronavirus-Infected Pneumonia in Wuhan, China. JAMA. 2020 Mar 17;323(11):1061-9. DOI: 10.1001/jama.2020.1585

17. Liu J, Liu Y, Xiang P, Pu L, Xiong H, Li C, et al. Neu- trophil-to-lymphocyte ratio predicts critical illness patients with 2019 coronavirus disease in the early stage. J Transl Med. 2020 May 20;18(1):206. DOI: 10.1186/ s12967-020-02374-0

18. Muniyappa R, Gubbi S. COVID-19 pandemic, coronaviruses, and diabetes mellitus. Am J Physiol Endocrinol Metab. 2020;318:E736-E741. DOI: 10.1152/ajpendo.00124.2020

19. Pradhan A, Olsson PE. Sex differences in severity and mortality from COVID-19: are males more vulnerable? Biol Sex Differ. 2020 Sep 18;11(1):53. DOI: 10.1186/ s13293-020-00330-7

20. Wong RS, Wu A, To KF, Lee N, Lam CWK, Wong $\mathrm{CK}$, et al. Haematological manifestations in patients with severe acute respiratory syndrome: retrospective analysis. BMJ 2003; 326: 1358-62. DOI: 10.1136/ bmj.326.7403.1358

21. Panesar, NS. What caused lymphopenia in SARS and how reliable is the lymphokine status in glucocorticoid-treated patients? Med Hypotheses 2008; 71: 298301. DOI: 10.1016/j.mehy.2008.03.019

22. Yang M, Li CK, Li K, Hon KL, Ng MH, Chan PK, et al. Hematological findings in SARS patients and possible mechanisms (review). Int J Mol Med 2004;14:311-5. DOI: 10.3892/ijmm.14.2.311 